\title{
Parabolic problems with data measure
}

\author{
Mohammmed Kbiri Alaoui
}

Correspondence: mka_la@yahoofr Department of Mathematics, King Khalid University, P.O. Box 9004, Abha, Kingdom of Saudi Arabia

\section{Abstract}

The article deals with the existence of solutions of some unilateral problems in the Orlicz-Sobolev spaces framework when the right-hand side is a Radon measure. Mathematics Subject Classification: 35K86.

Keywords: unilateral problem, radon measure, Orlicz-Sobolev spaces

\section{Introduction}

We deal with boundary value problems

$$
\begin{cases}u \geq \psi & \text { a.e. in } Q=\Omega \times[0, T] \\ \frac{\partial u}{\partial t}+\mathcal{A}(u)=\mu & \text { in } Q, \\ u=0 & \text { on } \partial Q=\partial \Omega \times[0, T] \\ u(x, 0)=u_{0}(x) & \text { in } \Omega,\end{cases}
$$

where

$$
\mathcal{A}(u)=-\operatorname{div}(a(., t, u, \nabla u)),
$$

$T>0$ and $\Omega$ is a bounded domain of $\mathbf{R}^{N}$, with the segment property. $a: \Omega \times \mathbf{R} \times \mathbf{R}^{N}$ $\rightarrow \mathbf{R}^{N}$ is a Carathéodory function (that is, measurable with respect to $x$ in $\Omega$ for every $(t, s, \xi)$ in $\mathbf{R} \times \mathbf{R} \times \mathbf{R}^{N}$, and continuous with respect to $(s, \xi)$ in $\mathbf{R} \times \mathbf{R}^{N}$ for almost every $x$ in $\Omega$ ) such that for all $\xi, \xi^{*} \in \mathbf{R}^{N}, \xi \neq \xi^{*}$,

$$
\begin{aligned}
& a(x, t, s, \xi) \xi \geq \alpha M(|\xi|) \\
& {\left[a(x, t, s, \xi)-a\left(x, t, s, \xi^{*}\right)\right]\left[\xi-\xi^{*}\right]>0,} \\
& |a(x, t, s, \xi)| \leq c(x, t)+k_{1} \bar{P}^{-1} M\left(k_{2}|s|\right)+k_{3} \bar{M}^{-1} M\left(k_{4}|\xi|\right),
\end{aligned}
$$

where $c(x, t)$ belongs to $E_{\bar{M}}(Q), c \geq 0, P$ is an $N$-function such that $P \ll M$ and $k_{i}(i$ $=1,2,3,4)$ belongs to $\mathbf{R}^{+}$and $\alpha$ to $\mathbf{R}_{*}^{+}$.

$$
\begin{aligned}
& \mu \in M_{b}^{+}(Q), \quad u_{0} \in M_{b}^{+}(\Omega), \\
& \psi \in L^{\infty}(\Omega) \cap W_{0}^{1} E_{M}(\Omega) .
\end{aligned}
$$

There have obviously been many previous studies on nonlinear differential equations with nonsmooth coefficients and measures as data. The special case was cited in the references (see [1,2]).

(c) 2011 Alaoui; licensee Springer. This is an Open Access article distributed under the terms of the Creative Commons Attribution License (http://creativecommons.org/licenses/by/2.0), which permits unrestricted use, distribution, and reproduction in any medium, provided the original work is properly cited. 
It is noteworthy that the articles mentioned above differ in significant way, in the terms of the structure of the equations and data. In [1], when $f \in L^{1}\left(0, T ; L^{1}(\Omega)\right)$ and $u_{0}$ $\in L^{1}(\Omega)$. The authors have shown the existence of solutions $u$ of the corresponding equation of the problem $(\mathcal{P}), u \in L^{q}\left(0, T ; W_{0}^{1, q}(\Omega)\right)$ for every $q$ such that $q<p-\frac{N}{N+1}$ which is more restrictive than the one given in the elliptic case $\left(q<\frac{N_{(p-1)}}{N-1}\right)$.

In this article, we are interested with an obstacle parabolic problem with measure as data. We give an improved regularity result of the study of Boccardo et al. [1].

In [1], the authors have shown the existence of a weak solutions for the corresponding equation, the function $a(x, t, s, \xi)$ was assumed to satisfy a polynomial growth condition with respect to $u$ and $\nabla u$. When trying to relax this restriction on the function $a(., \mathrm{s}, \xi)$, we are led to replace the space $L^{p}\left(0, T ; W^{1, p}(\Omega)\right)$ by an inhomogeneous Sobolev space $W^{1, x} L_{M}$ built from an Orlicz space $L_{M}$ instead of $L^{p}$, where the $N$-function $M$ which defines $L_{M}$ is related to the actual growth of the Carathéodory's function.

For simplicity, one can suppose that there exist $\alpha>0, \beta>0$ such that

$$
a(x, t, u, \nabla u)=a(x, t, u) \frac{M(|\nabla u|)}{|\nabla u|^{2}} \nabla u \text { and } \alpha \leq-a(x, t, s) \mid \leq \beta .
$$

\section{Preliminaries}

Let $M: \mathbf{R}^{+} \rightarrow \mathbf{R}^{+}$be an $N$-function, i.e. $M$ is continuous, convex, with $M(t)>0$ for $t>0, \frac{M(t)}{t} \rightarrow 0$ as $t \rightarrow 0$ and $\frac{M(t)}{t} \rightarrow \infty$ as $t \rightarrow \infty$. Equivalently, $M$ admits the representation: $M(t)=\int_{0}^{t} a(\tau) d \tau$ where $a: \mathbf{R}^{+} \rightarrow \mathbf{R}^{+}$is non-decreasing, right continuous, with $a(0)=0, a(t)>0$ for $t>0$ and $a(t) \rightarrow \infty$ as $t \rightarrow \infty$. The $N$-function $\bar{M}$ conjugate to $M$ is defined by $\bar{M}(t)=\int_{0}^{t} \bar{a}(\tau) d \tau$, where $\bar{a}: \mathbf{R}^{+} \rightarrow \mathbf{R}^{+}$is given by $\bar{a}(t)=\sup \{s: a(s) \leq t\}($ see $[3,4])$.

The $N$-function $M$ is said to satisfy the $\Delta_{2}$ condition if, for some $k>0$ :

$$
M(2 t) \leq k M(T) \text { for all } t \geq 0,
$$

when this inequality holds only for $t \geq t_{0}>0, M$ is said to satisfy the $\Delta_{2}$ condition near infinity.

Let $P$ and $Q$ be two $N$-functions. $P \ll Q$ means that $P$ grows essentially less rapidly than $Q$; i.e., for each $\varepsilon>0$,

$$
\frac{P(t)}{Q(\varepsilon t)} \rightarrow 0 \quad \text { as } t \rightarrow \infty \text {. }
$$

Let $\Omega$ be an open subset of $\mathbf{R}^{N}$. The Orlicz class $\mathcal{L}_{M}(\Omega)$ (resp. the Orlicz space $L_{m}$ $(\Omega)$ ) is defined as the set of (equivalence classes of) real-valued measurable functions $u$ on $\Omega$ such that

$$
\int_{\Omega} M(u(x)) d x<+\infty \quad\left(\text { resp. } \int_{\Omega} M\left(\frac{u(x)}{\lambda}\right) d x<+\infty \text { for some } \lambda>0\right) .
$$


Note that $L_{M}(\Omega)$ is a Banach space under the norm $\|u\|_{M, \Omega}=\inf \left\{\lambda>0: \int_{\Omega} M\left(\frac{u(x)}{\lambda}\right) d x \leq 1\right\}$ and $\mathcal{L}_{M}(\Omega)$ is a convex subset of $L_{M}(\Omega)$. The closure in $L_{M}(\Omega)$ of the set of bounded measurable functions with compact support in $\bar{\Omega}$ is denoted by $E_{M}(\Omega)$. The equality $E_{M}(\Omega)=L_{M}(\Omega)$ holds if and only if $M$ satisfies the $\Delta_{2}$ condition, for all $t$ or for $t$ large according to whether $\Omega$ has infinite measure or not.

The dual of $E_{M}(\Omega)$ can be identified with $L_{\bar{M}}(\Omega)$ by means of the pairing $\int_{\Omega} u(x) v(x) d x$, and the dual norm on $L_{\bar{M}}(\Omega)$ is equivalent to $\|\cdot\|_{\bar{M}, \Omega}$. The space $L_{M}(\Omega)$ is reflexive if and only if $M$ and $\bar{M}$ satisfy the $\Delta_{2}$ condition, for all $t$ or for $t$ large, according to whether $\Omega$ has infinite measure or not.

We now turn to the Orlicz-Sobolev space. $W^{1} L_{M}(\Omega)$ (resp. $W^{1} E_{M}(\Omega)$ ) is the space of all functions $u$ such that $u$ and its distributional derivatives up to order 1 lie in $L_{M}(\Omega)$ (resp. $\left.E_{M}(\Omega)\right)$. This is a Banach space under the norm $\|u\|_{1, M, \Omega}=\sum_{|\alpha| \leq 1}\left\|D^{\alpha} u\right\|_{M, \Omega}$. Thus, $W^{1} L_{M}(\Omega)$ and $W^{1} E_{M}(\Omega)$ can be identified with subspaces of the product of $N+$ 1 copies of $L_{M}(\Omega)$. Denoting this product by $\Pi L_{M}$, we will use the weak topologies $\sigma\left(\prod L_{M}, \prod E_{\bar{M}}\right)$ and $\sigma\left(\prod L_{M}, \prod L_{\bar{M}}\right)$. The space $W_{0}^{1} E_{M}(\Omega)$ is defined as the (norm) closure of the Schwartz space $\mathcal{D}(\Omega)$ in $W^{1} E_{M}(\Omega)$ and the space $W_{0}^{1} L_{M}(\Omega)$ as the $\sigma\left(\prod L_{M}, \prod E_{\bar{M}}\right)$ closure of $\mathcal{D}(\Omega)$ in $W^{1} L_{M}(\Omega)$. We say that $u_{n}$ converges to $u$ for the modular convergence in $W^{1} L_{M}(\Omega)$ if for some $\lambda>0, \int_{\Omega} M\left(\frac{D^{\alpha} u_{n}-D^{\alpha} u}{\lambda}\right) d x \rightarrow 0$ for all $|\alpha| \leq 1$. This implies convergence for $\sigma\left(\prod L_{M}, \prod L_{\bar{M}}\right)$. If $M$ satisfies the $\Delta_{2}$ condition on $\mathbf{R}^{+}$(near infinity only when $\Omega$ has finite measure), then modular convergence coincides with norm convergence.

Let $W^{-1} L_{\bar{M}}(\Omega)$ (resp. $W^{-1} E_{\bar{M}}(\Omega)$ ) denote the space of distributions on $\Omega$ which can be written as sums of derivatives of order $\leq 1$ of functions in $L_{\bar{M}}(\Omega)$ (resp. $\left.E_{\bar{M}}(\Omega)\right)$. It is a Banach space under the usual quotient norm.

If the open set $\Omega$ has the segment property, then the space $\mathcal{D}(\Omega)$ is dense in $W_{0}^{1} L_{M}(\Omega)$ for the modular convergence and for the topology $\sigma\left(\prod L_{M}, \prod L_{\bar{M}}\right)(\mathrm{cf}$. $[5,6])$. Consequently, the action of a distribution in $W^{-1} L_{\bar{M}}(\Omega)$ on an element of $W_{0}^{1} L_{M}(\Omega)$ is well defined.

For $k>0, s \in \mathbf{R}$, we define the truncation at height $k, T_{k}(s)=\left[k-(k-|s|)_{+}\right] \operatorname{sign}(s)$.

The following abstract lemmas will be applied to the truncation operators.

Lemma 2.1 [7] Let $F: \mathbf{R} \rightarrow \mathbf{R}$ be uniformly lipschitzian, with $F(0)=0$. Let $M$ be an $N$-function and let $u \in W_{0}^{1} L_{M}(\Omega)\left(\right.$ resp. $\left.W_{0}^{1} E_{M}(\Omega)\right)$.

Then $F(u) \in W_{0}^{1} L_{M}(\Omega)\left(\right.$ resp. $\left.W_{0}^{1} E_{M}(\Omega)\right)$. Moreover, if the set of discontinuity points of $F^{\prime}$ is finite, then

$$
\frac{\partial}{\partial x_{i}} F(u)= \begin{cases}F^{\prime}(u) \frac{\partial u}{\partial x_{i}} & \text { a.e. in }\{x \in \Omega: u(x) \notin D\} \\ 0 & \text { a.e. in }\{x \in \Omega: u(x) \in D\}\end{cases}
$$

Let $\Omega$ be a bounded open subset of $\mathbf{R}^{N}, T>0$ and set $\left.Q=\Omega \times\right] 0, T[$. Let $m \geq 1$ be an integer and let $M$ be an $N$-function. For each $\alpha \in \mathbf{I N}^{N}$, denote by $D_{x}^{\alpha}$ the distributional derivative on $Q$ of order $\alpha$ with respect to the variable $x \in \mathbf{R}^{N}$. The 
inhomogeneous Orlicz-Sobolev spaces are defined as follows $W^{m, x} L_{M}(Q)=\left\{u \in L_{M}(Q): D_{x}^{\alpha} u \in L_{M}(Q) \forall|\alpha| \leq m\right\} W^{m, x} E_{M}(Q)=\left\{u \in E_{M}(Q):\right.$ $\left.D_{x}^{\alpha} u \in E_{M}(Q) \forall|\alpha| \leq m\right\}$

The last space is a subspace of the first one, and both are Banach spaces under the norm $\|u\|=\sum_{|\alpha| \leq m}\left\|D_{x}^{\alpha} u\right\|_{M, Q}$. We can easily show that they form a complementary system when $\Omega$ satisfies the segment property. These spaces are considered as subspaces of the product space $\Pi L_{m}(Q)$ which have as many copies as there are $\alpha$-order derivatives, $|\alpha| \leq m$. We shall also consider the weak topologies $\sigma\left(\prod L_{M}, \prod E_{\bar{M}}\right)$ and $\sigma\left(\prod L_{M}, \prod L_{\bar{M}}\right)$. If $u \in W^{m, x} L_{M}(Q)$, then the function : $t \mapsto u(t)=u(t,$.$) is defined on$ $[0, T]$ with values in $W^{m} L_{M}(\Omega)$. If, further, $u \in W^{m, x} E_{M}(Q)$ then the concerned function is a $W^{m} E_{M}(\Omega)$-valued and is strongly measurable. Furthermore, the following imbedding holds: $W^{m, x} E_{M}(Q) \subset L^{1}\left(0, T ; W^{m} E_{M}(\Omega)\right)$. The space $W^{m, x} L_{M}(Q)$ is not in general separable, if $u \in W^{m, x} L_{M}(Q)$, we cannot conclude that the function $u(t)$ is measurable on $[0, T]$. However, the scalar function $t \mapsto\|u(t)\|_{M, \Omega}$, is in $L^{1}(0, T)$. The space $W_{0}^{m, x} E_{M}(Q)$ is defined as the (norm) closure in $W^{m, x} E_{M}(Q)$ of $\mathcal{D}(\Omega)$. We can easily show as in [6] that when $\Omega$ has the segment property, then each element $u$ of the closure of $\mathcal{D}(\Omega)$ with respect of the weak * topology $\sigma\left(\prod L_{M}, \prod E_{\bar{M}}\right)$ is limit, in $W^{m, x} L_{M}$ $(Q)$, of some subsequence $\left(u_{i}\right) \subset \mathcal{D}(Q)$ for the modular convergence; i.e., there exists $\lambda$ $>0$ such that for all $|\alpha| \leq m$,

$$
\int_{Q} M\left(\frac{D_{x}^{\alpha} u_{i}-D_{x}^{\alpha} u}{\lambda}\right) d x d t \rightarrow 0 \text { as } i \rightarrow \infty,
$$

this implies that $\left(u_{i}\right)$ converges to $u$ in $W^{m, x} L_{M}(Q)$ for the weak topology $\sigma\left(\prod L_{M}, \prod L_{\bar{M}}\right)$. Consequently, $\overline{\mathcal{D}(Q)}^{\sigma\left(\prod L_{M}, \prod E_{\bar{M}}\right)}=\overline{\mathcal{D}(Q)}^{\sigma\left(\prod L_{M}, \prod L_{\bar{M}}\right)}$, and this space will be denoted by $W_{0}^{m, x} L_{M}(Q)$.

Furthermore, $W_{0}^{m, x} E_{M}(Q)=W_{0}^{m, x} L_{M}(Q) \cap \prod E_{M}$. Poincaré's inequality also holds in $W_{0}^{m, x} L_{M}(Q)$, i.e., there is a constant $C>0$ such that for all $u \in W_{0}^{m, x} L_{M}(Q)$ one has $\sum_{|\alpha| \leq m}\left\|D_{x}^{\alpha} u\right\|_{M, Q} \leq C \sum_{|\alpha|=m}\left\|D_{x}^{\alpha} u\right\|_{M, Q}$. Thus both sides of the last inequality are equivalent norms on $W_{0}^{m, x} L_{M}(Q)$. We have then the following complementary system:

$$
\left(\begin{array}{ll}
W_{0}^{m, x} L_{M}(Q) & F \\
W_{0}^{m, x} E_{M}(Q) & F_{0}
\end{array}\right)
$$

$F$ being the dual space of $W_{0}^{m, x} E_{M}(Q)$. It is also, except for an isomorphism, the quotient of $\prod L_{\bar{M}}$ by the polar set $W_{0}^{m, x} E_{M}(Q) \perp$, and will be denoted by $F=W^{-m, x} L_{\bar{M}}(Q)$, and it is shown that $W^{-m, x} L_{\bar{M}}(Q)=\left\{f=\sum_{|\alpha| \leq m} D_{x}^{\alpha} f_{\alpha}: f_{\alpha} \in L_{\bar{M}}(Q)\right\}$. This space will be equipped with the usual quotient norm $\|f\|=\inf \sum_{|\alpha| \leq m}\left\|f_{\alpha}\right\|_{\bar{M}, Q}$ where the infimum is taken on all possible decompositions $f=\sum_{|\alpha| \leq m} D_{x}^{\alpha} f_{\alpha \prime}, \quad f_{\alpha} \in L_{\bar{M}}(Q)$. The space $F_{0}$ is then given by $F_{0}=\left\{f=\sum_{|\alpha| \leq m} D_{x}^{\alpha} f_{\alpha}: f_{\alpha} \in E_{\bar{M}}(Q)\right\}$ and is denoted by $F_{0}=W^{-m, x} E_{\bar{M}}(Q)$.

We can easily check, using Lemma 4.4 of [6], that each uniformly lipschitzian mapping $F$, with $F(0)=0$, acts in inhomogeneous Orlicz-Sobolev spaces of order $1: W^{1, x} L_{M}$ $(Q)$ and $W_{0}^{1, x} L_{M}(Q)$. 


\section{Main results}

First, we give the following results which will be used in our main result.

\subsection{Useful results}

Hereafter, we denote by $\mathcal{X}_{N}$ the real number defined by $\mathcal{X}_{N}=N C_{N}^{1 / N}, C_{N}$ is the measure of the unit ball of $\mathbf{R}^{N}$, and for a fixed $t \in[0, T]$, we denote $\mu(\theta)=$ meas $\{(x, t): \mid u$ $(x, t) \mid>\theta\}$.

Lemma 3.1 [8]Let $u \in W_{0}^{1, x} L_{M}(Q)$, and let fixed $t \in[0, T]$, then we have

$$
-\mu^{\prime}(\theta) \geq-\frac{1}{\mathcal{X}_{N} \mu(\theta)^{1-\frac{1}{N}}} \mathcal{S}\left(-\frac{1}{\mathcal{X}_{N} \mu(\theta)^{1-\frac{1}{N}}} \frac{d}{d \theta} \int_{\{|u|>\theta\}} M(|\nabla u|) d x\right), \forall \theta>0
$$

and where Sis defined by

$$
\frac{1}{\mathcal{S}(s)}=\sup \{t: B(t) \leq s\}, \quad B(s)=\frac{M(s)}{s} .
$$

Lemma 3.2 Under the hypotheses (1.1)-(1.3), if $f, u_{0}$ are regular functions and $f, u_{0} \geq$ 0 , then there exists at least one positive weak solution of the problem

$$
\left\{\begin{array}{l}
\frac{\partial u}{\partial t}+\mathcal{A}(u)=f \text { in } \quad Q \\
u=0 \quad \text { on } \partial Q \\
u(x, 0)=u_{0}(x) \text { in } \quad \Omega .
\end{array}\right.
$$

such that

$$
\frac{\partial u}{\partial t} \geq 0, \quad \text { a.e. } t \in(0, T) .
$$

\section{Proof}

Let $u$ be a continuous function, we say that $u$ satisfies (*) if: there exists a continuous and increasing function $\beta$ such that $\|u(t)-u(s)\|_{2} \leq \beta\left(\| u_{0}||_{2}\right)|t-s|$, where $u_{0}(x)=u(x$, $0)$.

$$
\text { Let } X:=\left\{u \in W_{0}^{1, x} L_{M}(Q) \cap L^{2}(Q) \text { s.t. } u \text { satisfies }(*) \text { and } \frac{d u}{d t} \in L^{\infty}\left(0, T, L^{2}(\Omega)\right)\right\} \text {. }
$$

Let us consider the set $\mathcal{C}=\left\{v \in X: v(t) \in C, \quad \frac{\partial v}{\partial t} \geq 0\right.$ a.e. $\left.t \in(0, T)\right\}$, where $C$ is a closed convex of $W_{0}^{1} L_{M}(\Omega)$. It is easy to see that $\mathcal{C}$ is a closed convex (since all its elements satisfy (*)).

We claim that the problem

$$
\begin{cases}u \in \mathcal{C} \cap L^{2}(Q) \\ \frac{\partial u}{\partial t}+\mathcal{A}(u)=f \text { in } Q \\ u=0 \quad \text { on } \partial Q \\ u(x, 0)=u_{0} \quad \text { in } \Omega\end{cases}
$$

has a weak solution which is unique in the sense defined in [9]. 
Indeed, let us consider the approximate problem

$$
\left\{\begin{array}{lr}
\frac{\partial u_{n}}{\partial t}+\mathcal{A}\left(u_{n}\right)+n T_{n}\left(\Phi\left(u_{n}\right)\right)= & f \text { in } \Omega, \\
u_{n}(., 0)=u_{0} & \text { in } \Omega .
\end{array}\right.
$$

where the functional $\Phi$ is defined by $\Phi: X \rightarrow \mathbf{R} \cup\{+\infty\}$ such that

$$
\Phi(v):= \begin{cases}0 & \text { if } v \in \mathcal{C} \\ +\infty & \text { otherwise }\end{cases}
$$

The existence of such $u_{n} \in X$ was ensured by Kacur et al. [10].

Following the same proof as in [9], we can prove the existence of a solution $u$ of the problem $\left(E^{\prime}\right)$ as limit of $u_{n}$ (for more details see [9]).

Lemma 3.3 Let $v \in W_{0}^{1, x} L_{M}(Q)$ such that $\frac{\partial v}{\partial t} \in W^{-1, x} L_{\bar{M}}(Q)+L^{1}(Q)$ and $v \geq \psi, \psi \in L^{\infty}(\Omega) \cap W_{0}^{1} E_{M}(\Omega)$.

Then, there exists a smooth function $\left(v_{j}\right)$ such that

$$
v_{j} \geq \psi,
$$

$v_{j} \rightarrow v$ for the modular convergence in $W_{0}^{1, x} L_{M}(Q)$,

$\frac{\partial v_{i}}{\partial t} \rightarrow \frac{\partial v}{\partial t}$ for the modular convergence in $W^{-1, x} L_{\bar{M}}(Q)+L^{1}(Q)$.

For the proof, we use the same technique as in [11] in the parabolic case.

\subsection{Existence result}

Let $M$ be a fixed $N$-function, we define $K$ as the set of $N$-function $D$ satisfying the following conditions:

i) $M\left(D^{-1}(s)\right)$ is a convex function,

ii) $\int_{0} D o B^{-1}\left(\frac{1}{r^{1-1 / N}}\right) d r<+\infty, B(t)=\frac{M(t)}{t}$,

iii) There exists an $N$-function $H$ such that $H o \bar{M}^{-1} o M \leq D$ and $\bar{H} \leq D$ near infinity.

Theorem 3.1 Under the hypotheses (1.1)-(1.5), The problem (P) has at least one solution $u$ in the following sense:

$$
\left\{\begin{array}{l}
u \geq \psi \text { a.e. in } Q \\
T_{k}(u) \in W_{0}^{1, x} L_{M}(Q), u \in W_{0}^{1, x} L_{D}(Q) \quad \forall D \in K \\
-\int_{Q} u \frac{\partial \varphi}{\partial t}+\int_{Q} a(., u, \nabla u) \nabla \varphi d x d t-\int_{\Omega} \varphi d u_{0}=\int_{Q} \varphi d \mu
\end{array}\right.
$$

for all $\phi \in D\left(\mathbf{R}^{N+1}\right)$ which are zero in a neighborhood of $(0, T) \times \partial \Omega$ and $\{T\} \times \Omega$.

Remark 3.1 (1) If $\psi=-\infty$ in the problem (P), then the above theorem gives the same regularity as in the elliptic case.

(2) An improved regularity is reached for all $\mathrm{N}$-function satisfying the conditions (i)(ii)-(iii).

$$
\text { For example, } u \in W_{0}^{1, x} L_{D}(Q), D(t)=\frac{t^{q}}{\log ^{\sigma}(e+t)} \text {, for all } q<\frac{N(p-1)}{N-1}, \sigma>1 \text {. }
$$

In the sequel and throughout the article, we will omit for simplicity the dependence on $x$ and $t$ in the function $a(x, t, s, \xi)$ and denote $\epsilon(n, j, \mu, s, m)$ all quantities (possibly different) such that 


$$
\lim _{m \rightarrow \infty} \lim _{s \rightarrow \infty} \lim _{\mu \rightarrow \infty} \lim _{j \rightarrow \infty} \lim _{n \rightarrow \infty} \varepsilon(n, j, \mu, s, m)=0,
$$

and this will be in the order in which the parameters we use will tend to infinity, that is, first $n$, then $j, \mu, s$, and finally $m$. Similarly, we will write only $\epsilon(n)$, or $\epsilon(n, j), \ldots$ to mean that the limits are made only on the specified parameters.

\subsubsection{A sequence of approximating problems}

Let $\left(f_{n}\right)$ be a sequence in $D(Q)$ which is bounded in $L^{1}(Q)$ and converge to $\mu$ in $M_{b}(Q)$.

Let $\left(u_{0}^{n}\right)$ be a sequence in $D(\Omega)$ which is bounded in $L^{1}(\Omega)$ and converge to $u_{0}$ in $M_{b}$ $(\Omega)$.

We define the following problems approximating the original $(P)$ :

$$
\begin{cases}\frac{\partial u_{n}}{\partial t}+\mathcal{A}\left(u_{n}\right)-n T_{n}\left(\left(u_{n}-\psi\right)^{-}\right)=f_{n} & \text { in } Q \\ u_{n}=0 & \text { on } \partial Q \\ u_{n}(., 0)=u_{0}^{n} & \text { in } \Omega .\end{cases}
$$

Lemma 3.4 Under the hypotheses (1.1)-(1.3), there exists at least one solution $u_{n}$ of the problem $\left(P_{n}\right)$ such that $\frac{\partial u_{n}}{\partial t} \geq 0$ a.e. in $Q$.

For the proof see Lemma 3.2.

\subsubsection{A priori estimates}

Lemma 3.5 There exists a subsequence of $\left(u_{n}\right)$ (also denoted $\left(u_{n}\right)$ ), there exists a measurable function $u$ such that:

$$
\begin{aligned}
& u \geq \psi, T_{k}(u) \in W_{0}^{1, x} L_{M}(Q) \text { for all } k>0 \\
& u_{n} \rightarrow u \text { weakly in } W_{0}^{1, x} L_{D}(Q) \text { for all } D \in K .
\end{aligned}
$$

Proof:

Recall that $u_{n} \geq 0$ since $f_{n} \geq 0$.

Let $h>0$ and consider the following test function $v=T_{h}\left(u_{n}-T_{k}\left(u_{n}\right)\right)$ in $\left(P_{n}\right)$, we obtain

$$
\ll \frac{\partial u_{n}}{\partial t}, v \gg+\alpha \int_{\left\{k<\left|u_{n}\right| \leq k+h\right\}} M\left(\left|\nabla u_{n}\right|\right) d x d t-n \int_{Q} T_{n}\left(\left(u_{n}-\psi\right)^{-}\right) v d x d t \leq \int_{Q} f_{n} v d x d t
$$

We have

$$
\ll \frac{\partial u_{n}}{\partial t}, T_{h}\left(u_{n}-T_{k}\left(u_{n}\right)\right) \gg=\int_{\Omega} \int_{0}^{u_{n}(x, T)} T_{h}\left(s-T_{k}(s)\right)-\int_{\Omega} \int_{0}^{u_{0}^{n}} T_{h}\left(s-T_{k}(s)\right) .
$$

So,

$$
-\int_{Q} n T_{n}\left(\left(u_{n}-\psi\right)^{-}\right) \frac{T_{h}\left(u_{n}-T_{k}\left(u_{n}\right)\right)}{h} d x d t \leq C .
$$

Now, let us fix $k>\|\psi\|_{\infty}$, we deduce the fact that: $n T_{n}\left(u_{n}-\psi\right)\left(u_{n}-k\right) \mathcal{X}_{\left\{u_{n} \leq \psi\right\}} \mathcal{X}_{\left\{k<u_{n} \leq k+h\right\}} \geq 0$.

Let $h$ to tend to zero, one has

$$
n \int_{Q} T_{n}\left(\left(u_{n}-\psi\right)^{-}\right) d x d t \leq C .
$$


Let us use as test function in $\left(P_{n}\right), v=T_{k}\left(u_{n}\right)$, then as above, we obtain

$$
\int_{Q} M\left(\left|\nabla T_{k}\left(u_{n}\right)\right|\right) \leq C_{1} k
$$

Then $\left(T_{k}\left(u_{n}\right)_{n}\right)$ is bounded in $W_{0}^{1, x} L_{M}(Q)$, and then there exist some $\omega_{k} \in W_{0}^{1, x} L_{M}(Q)$ such that

$T_{k}\left(u_{n}\right) \rightarrow \omega_{k}$, weakly in $W_{0}^{1, x} L_{M}(Q)$ for $\sigma\left(\prod L_{M}, \prod E_{\bar{M}}\right)$, strongly in $E_{M}(Q)$ and a.e in Q.

Let consider the $C^{2}$ function defined by

$$
\mu_{k}(s) \begin{cases}s & |s| \leq k / 2 \\ k \operatorname{sign}(s) & |s| \geq k\end{cases}
$$

Multiplying the approximating equation by $\eta_{k}^{\prime}\left(u_{n}\right)$, we get $\frac{\partial \eta_{k}\left(u_{n}\right)}{\partial t}-\operatorname{div}\left(a\left(., u_{n}, \nabla u_{n}\right) \eta_{k}^{\prime}\left(u_{n}\right)\right)+a\left(., u_{n} \nabla u_{n}\right) n_{k}^{\prime \prime}\left(u_{n}\right)=f_{n} \eta_{k}^{\prime}\left(u_{n}\right)+n\left(T_{n}\left(\left(u_{n}-\psi\right)^{-}\right)\right) \eta_{k}^{\prime}\left(u_{n}\right)$ in the distributions sense. We deduce then that $\eta_{k}\left(u_{n}\right)$ being bounded in $W_{0}^{1, x} L_{M}(Q)$ and $\frac{\partial \eta_{k}\left(u_{n}\right)}{\partial t}$ in $W^{-1, x} L_{\bar{M}}(Q)+L^{1}(Q)$. By Corollary 1 of [12], $\eta_{k}\left(u_{n}\right)$ is compact in $L^{1}(Q)$.

Following the same way as in [2], we obtain for every $k>0$,

$$
T_{k}\left(u_{n}\right) \rightarrow T_{k}(u) \text {, weakly in } W_{0}^{1, x} L_{M}(Q) \text { for } \sigma\left(\prod L_{M}, \prod E_{\bar{M}}\right) \text {, strongly in } L^{1}(Q) \text { and a.e in } Q \text {. }
$$

Using now the estimation (3.1) and Fatou's lemma to obtain

$$
(u-\psi)^{-}=0 \text { and so }, u \geq \psi \text {. }
$$

Let fixed a $t \in[0, T]$. We argue now as for the elliptic case, the problem becomes:

$$
\frac{\partial u_{n}}{\partial t}-\operatorname{div}\left(a\left(., u_{n}, \nabla u_{n}\right)\right)=f_{n}+n T_{n}\left(\left(u_{n}-\psi\right)^{-}\right) \quad \text { in } \Omega . \quad\left(P_{n}^{\prime}\right)
$$

We denote $g_{n}:=n T_{n}\left(\left(u_{n}-\psi\right)^{-}\right)$.

Let $\phi$ be a truncation defined by

$$
\varphi(\xi)= \begin{cases}0 & 0 \leq \xi \leq \theta \\ \frac{1}{h}(\xi-t) & \theta<\xi<\theta+h \\ 1 & \xi \geq \theta+h \\ -\varphi(-\xi) & \xi<0\end{cases}
$$

for all $\theta, h>0$.

Using $v=\phi\left(u_{n}\right)$ as a test function in the approximate elliptic problem $\left(P_{n}^{\prime}\right)$, we obtain by using the same technique as in [8]

$$
-\frac{d}{d \theta} \int_{\left\{\left|u_{n}\right|>\theta\right\}} M\left(\left|\nabla u_{n}\right|\right) d x \leq C \int_{\left\{\left|u_{n}\right| \geq \theta\right\}}\left(f_{n}+g_{n}-\frac{\partial u_{n}}{\partial t}\right) d x .
$$

here and below $C$ denote positive constants not depending on $n$.

By using Lemma 3.1, we obtain (supposing $-\mu^{\prime}(\theta)>0$ which does not affect the proof) and following the same way as in [8], we have for $D \in K$, 


$$
-\frac{d}{d \theta} \int_{\left\{\left|u_{n}\right|>\theta\right\}} D\left(\left|\nabla u_{n}\right|\right) d x \leq\left(-\mu^{\prime}(\theta)\right) D o B^{-1}\left(\left(-\frac{1}{\mathcal{X}_{N \mu}(\theta){ }^{1-\frac{1}{N}}} \frac{d}{d \theta} \int_{\left\{\left|u_{n}\right|>\theta\right\}} M\left(\left|\nabla u_{n}\right| d x\right)\right)\right) .
$$

Let denote $k(t, s):=\int_{0}^{s} u_{n *}(t, \rho) d \rho$, then

$$
\frac{\partial k}{\partial t}(t, s)=\int_{0}^{s} \frac{\partial u_{n *}(t, \rho)}{\partial t} d \rho, \quad \int_{u_{n}>\theta} \frac{\partial u_{n}}{\partial t} d x=\frac{\partial k}{\partial t}(t, \mu(\theta)) .
$$

Using Lemma 3.1, denoting $F(t, \mu(\theta)):=\int_{0}^{\mu(\theta)}\left(f_{n *}+g_{n *}\right)(\rho) d \rho$ one has

$$
1 \leq \frac{-\mu^{\prime}(\theta)}{\mathcal{X}_{N} \mu(\theta)^{1-\frac{1}{N}}} B^{-1}\left(\frac{1}{\mathcal{X}_{N} \mu(\theta)^{1-\frac{1}{N}}}\left[F(t, \mu(\theta))-\frac{\partial k}{\partial t}(t, \mu(\theta))\right]\right) .
$$

Remark also that $F(t, s) \geq \frac{\partial k}{\partial t}(t, s)$ and using Lemma 3.2, we have $\left|\frac{\partial k}{\partial t}(t, s)\right| \leq F(t, s)$.

Combining the inequalities (3.5) and (3.6) we obtain,

$$
-\frac{d}{d \theta} \int_{\left\{\left|u_{n}\right|>\theta\right\}} D\left(\left|\nabla u_{n}\right|\right) d x \leq\left(-\mu^{\prime}(\theta)\right) D_{0} B^{-1}\left(-\frac{1}{\mathcal{X}_{N} \mu(\theta)^{1-\frac{1}{N}}}\left[F(t, \mu(\theta))-\frac{\partial k}{\partial t}(t, \mu(\theta))\right]\right) .
$$

and since the function $\theta \rightarrow \int_{\left\{\left|u_{n}\right|>\theta\right\}} D\left(\left|\nabla u_{n}\right|\right) d x$ is absolutely continuous, we get

$$
\begin{aligned}
\int_{\Omega} D\left(\left|\nabla u_{n}\right|\right) d x & =\int_{0}^{+\infty}\left(-\frac{d}{d \theta} \int_{\left\{\left|u_{n}\right|>\theta\right\}} D\left(\left|\nabla u_{n}\right|\right) d x\right) d t \\
& \leq \frac{1}{C^{\prime}} \int_{0}^{C^{\prime}|\Omega|} \operatorname{DoB}^{-1}\left(\left(\frac{C}{s^{1-1 / N}}\right)\right) d s(\text { using 3.1 and 3.7). }
\end{aligned}
$$

Then, the sequence $\left(u_{n}\right)$ is bounded in $W_{0}^{1, x} L_{D}(Q)$ and we deduce that $u \in W_{0}^{1, x} L_{D}(Q)$ for all $N$-function $D \in K$.

\subsection{Almost everywhere convergence of the gradients}

Lemma 3.6 The subsequence $\left(u_{n}\right)$ obtained in Lemma 3.5 satisfies:

$$
\nabla u_{n} \rightarrow \nabla u \text { a.e. in } Q \text {. }
$$

Proof:

Let $m>0, k>0$ such that $m>k$. Let $\rho_{m}$ be a truncation defined by

$$
\begin{gathered}
\rho m(s)= \begin{cases}1 & |s| \leq m, \\
m+1-|s| & m<|s|<m+1 \\
0 & |s| \geq m+1\end{cases} \\
R_{m}(s)=\int_{0}^{s} \rho_{m}(t) d t \text { and } \omega_{\mu, j}=T_{k}\left(v_{j}\right)_{\mu .}
\end{gathered}
$$

where $v_{j} \in D(Q)$ such that $v_{j} \geq \psi$ and $v_{j} \rightarrow T_{k}(u)$ with the modular convergence in $W_{0}^{1, x} L_{M}(Q)$ (for the existence of such function see [11] since $\psi \in L^{\infty}(\Omega) \cap W_{0}^{1} E_{M}(\Omega)$ ). 
$\omega_{\mu}$ is the mollifier function defined in Landes [13], the function $\omega_{\mu, j}$ have the following properties:

$$
\left\{\begin{array}{l}
\frac{\partial \omega_{\mu, j}}{\partial t}=\mu\left(T_{k}\left(v_{j}\right)-\omega_{\mu, j}\right), \omega_{\mu, j}(0)=0,\left|\omega_{\mu, j}\right| \leq k, \\
\omega_{\mu, j} \rightarrow T_{k}(u)_{\mu} \text { in } W_{0}^{1, x} L_{M}(Q) \text { for the modular convergence with respect to } j, \\
T_{k}(u)_{\mu} \rightarrow T_{k}(u) \text { in } W_{0}^{1, x} L_{M}(Q) \text { for the modular convergence with respect to } \mu .
\end{array}\right.
$$

Set $v=\left(T_{k}\left(u_{n}\right)-\omega_{\mu, j}\right) \rho_{m}\left(u_{n}\right)$ as test function, we have

$$
\begin{aligned}
& \ll \frac{\partial u_{n}}{\partial t}, v \gg \\
& +\int_{Q} a\left(., u_{n}, \nabla u_{n}\right)\left(\nabla T_{k}\left(u_{n}\right)-\nabla \omega_{\mu, j}^{i}\right) \rho_{m}\left(u_{n}\right) \\
& +\int_{Q} a\left(., u_{n}, \nabla u_{n}\right) \nabla u_{n}\left(T_{k}\left(u_{n}\right)-\omega_{\mu, j}\right) \rho_{m}^{\prime}\left(u_{n}\right) \\
& =\int_{Q} f_{n} v d x d t+n \int_{Q} T_{n}\left(\left(u_{n}-\psi\right)^{-}\right) v d x d t \\
& =:(3)+(4) .
\end{aligned}
$$

Let us recall that for $u_{n} \in W_{0}^{1, x} L_{M}(Q)$, there exists a smooth function $u_{n \sigma}$ (see [14]) such that

$$
\begin{aligned}
& u_{n \sigma} \rightarrow u_{n} \text { for the modular convergence in } W_{0}^{1, x} L_{M}(Q), \\
& \frac{\partial u_{n \sigma}}{\partial t} \rightarrow \frac{\partial u_{n}}{\partial t} \text { for the modular convergence in } W^{-1, x} L_{\bar{M}}(Q)+L^{1}(Q) . \\
& \ll \frac{\partial u_{n}}{\partial t}, v \gg=\lim _{\sigma \rightarrow 0+} \int_{Q}\left(u_{n \sigma}\right)^{\prime}\left(T_{k}\left(u_{n \sigma}\right)-\omega_{\mu, j}\right) \rho_{m}\left(u_{n \sigma}\right) \\
& =\lim _{\sigma \rightarrow 0+}\left(\int_{Q}\left(R_{m}\left(u_{n \sigma}\right)-T_{k}\left(u_{n \sigma}\right)\right)^{\prime}\left(T_{k}\left(u_{n \sigma}\right)-\omega_{\mu, j}\right) d x d t+\int_{Q}\left(T_{k}\left(u_{n \sigma}\right)^{\prime}\left(T_{k}\left(u_{n \sigma}\right)-\omega_{\mu, j}\right) d x d t\right)\right. \\
& =\lim _{\sigma \rightarrow 0+}\left[\int_{\Omega}\left(R_{m}\left(u_{n \sigma}\right)-T_{k}\left(u_{n \sigma}\right)\right)\left(T_{k}\left(u_{n \sigma}\right)-\omega_{\mu, j}\right) d x\right]_{0}^{T} \\
& -\int_{Q}\left(R_{m}\left(u_{n \sigma}\right)-T_{k}\left(u_{n \sigma}\right)\right)\left(T_{k}\left(u_{n \sigma}\right)-\omega_{\mu, j}\right)^{\prime} d x d t \\
& +\int_{Q}\left(T_{k}\left(u_{n \sigma}\right)^{\prime}\left(T_{k}\left(u_{n \sigma}\right)-\omega_{\mu, j}\right) d x d t=: I_{1}+I_{2}+I_{3} .\right.
\end{aligned}
$$

Remark also that,

$$
\begin{aligned}
R_{m}\left(u_{n \sigma}\right) & \geq T_{k}\left(u_{n \sigma}\right) \text { if } u_{n \sigma}<k \text { and } R_{m}\left(u_{n \sigma}\right)>k=T_{k}\left(u_{n \sigma}\right) \geq\left|\omega_{\mu, j}\right| \text { if } u_{n \sigma} \geq k . \\
I_{1} & =\int_{\Omega}\left(R_{m}\left(u_{n \sigma}\right)(T)-T_{k}\left(u_{n \sigma}\right)(T)\right)\left(T_{k}\left(u_{n \sigma}\right)(T)-\omega_{\mu, j}(T)\right) d x \\
I_{1} & \geq \int_{u n \sigma(T) \leq k}\left(R_{m}\left(u_{n \sigma}\right)(T)-T_{k}\left(u_{n \sigma}\right)(T)\right)\left(T_{k}\left(u_{n \sigma}\right)(T)-\omega_{\mu, j}(T)\right) d x
\end{aligned}
$$

and it is easy to see that $\limsup _{\sigma \rightarrow 0+} I_{1} \geq \varepsilon(n, j, \mu)$.

Concerning $I_{2}$,

$$
\begin{aligned}
& I_{2}=-\int_{u_{n \sigma} \leq k}\left(R_{m}\left(u_{n \sigma}\right)-T_{k}\left(u_{n \sigma}\right)\right)\left(T_{k}\left(u_{n \sigma}\right)-\omega_{\mu, j}\right)^{\prime} d x d t+\int_{u_{n \sigma}>k}\left(R_{m}\left(u_{n \sigma}\right)-T_{k}\left(u_{n \sigma}\right)\right)\left(\omega_{\mu, j}\right)^{\prime} d x d t \\
& =: I_{2}^{1}+I_{2}^{2} .
\end{aligned}
$$

As in $I_{1}$, we obtain $I_{2}^{1} \geq \varepsilon(n, j, \mu)$, 
and

$$
I_{2}^{2}=\int_{u_{n \sigma}>k}\left(R_{m}\left(u_{n \sigma}\right)-T_{k}\left(u_{n \sigma}\right)\right)\left(\omega_{\mu, j}\right)^{\prime} d x d t \geq \mu \int_{u_{n \sigma}>k}\left(R_{m}\left(u_{n \sigma}\right)-T_{k}\left(u_{n \sigma}\right)\right)\left(T_{k}\left(v_{j}\right)-T_{k}\left(u_{n \sigma}\right)\right)^{\prime} d x d t
$$

thus by using the fact that $\left(R_{m}\left(u_{n \sigma}\right)-T_{k}\left(u_{n \sigma}\right)\right)\left(T_{k}\left(u_{n \sigma}\right)-\omega_{\mu, j}\right) \mathcal{X}_{u_{n \sigma}>k} \geq 0$.

So, $\limsup _{\sigma \rightarrow 0^{+}} I_{2}^{2} \geq \mu \int_{u_{n}>k}\left(R_{m}\left(u_{n}\right)-T_{k}\left(u_{n}\right)\right)\left(T_{k}\left(v_{j}\right)-T_{k}\left(u_{n}\right)\right)^{\prime} d x d t=\varepsilon(n, j)$.

About $I_{3}$,

$$
\begin{aligned}
I_{3} & =\int_{Q}\left(T_{k}\left(u_{n \sigma}\right)\right)^{\prime}\left(T_{k}\left(u_{n \sigma}\right)-\omega_{\mu, j}\right) d x d t \\
& =\int_{Q}\left(T_{k}\left(u_{n \sigma}\right)-\omega_{\mu, j}\right)^{\prime}\left(T_{k}\left(u_{n \sigma}\right)-\omega_{\mu, j}\right) d x d t+\int_{Q}\left(\omega_{\mu, j}\right)^{\prime}\left(T_{k}\left(u_{n \sigma}\right)-\omega_{\mu, j}\right) d x d t .
\end{aligned}
$$

Set $\Phi(s)=s^{2} / 2, \Phi \geq 0$, then

$$
\begin{aligned}
I_{3} & =\left[\int_{\Omega} \Phi\left(T_{k}\left(u_{n \sigma}\right)-\omega_{\mu, j}\right) d x\right]_{0}^{T}+\mu \int_{Q}\left(T_{k}\left(v_{j}\right)-\omega_{\mu, j}\right)\left(T_{k}\left(u_{n \sigma}\right)-\omega_{\mu, j}\right) d x d t \\
& \geq \varepsilon(n, j, \mu)+\mu \int_{Q}\left(T_{k}\left(v_{j}\right)-T_{k}\left(u_{n \sigma}\right)\right)\left(T_{k}\left(u_{n \sigma}\right)-\omega_{\mu, j}\right) d x d t\left(\text { as in } I_{2}\right) .
\end{aligned}
$$

So,

$$
\begin{aligned}
\limsup _{\sigma \rightarrow 0^{+}} I_{3} & \geq \varepsilon(n, j, \mu)+\mu \int_{Q}\left(T_{k}\left(v_{j}\right)-T_{k}\left(u_{n}\right)\right)\left(T_{k}\left(u_{n}\right)-\omega_{\mu, j}\right) d x d t \\
& =\varepsilon(n, j, \mu)+\mu \int_{Q}\left(T_{k}\left(v_{j}\right)-T_{k}(u)\right)\left(T_{k}(u)-\omega_{\mu, j}\right) d x d t+\varepsilon(n),
\end{aligned}
$$

and easily we deduce, $\underset{\sigma \rightarrow 0^{+}}{\limsup } I_{3} \geq \varepsilon(n, j, \mu)$.

Finally we conclude that: $\ll \frac{\partial u_{n}}{\partial t},\left(T_{k}\left(u_{n}\right)-\omega_{\mu, j}\right) \rho_{m}\left(u_{n}\right) \gg \geq \varepsilon(n, j, \mu)$.

We are interested now with the terms of (1)-(4).

About (1):

$$
\begin{aligned}
& \int_{Q} a\left(., u_{n}, \nabla u_{n}\right)\left(\nabla T_{k}\left(u_{n}\right)-\nabla \omega_{\mu, j}\right) \rho_{m}\left(u_{n}\right) d x d t \\
& =\int_{u_{n} \leq k} a\left(., u_{n}, \nabla u_{n}\right)\left(\nabla T_{k}\left(u_{n}\right)-\nabla \omega_{\mu, j}\right) \rho_{m}\left(u_{n}\right) d x d t+\int_{u_{n}>k} a\left(., u_{n}, \nabla u_{n}\right)\left(\nabla T_{k}\left(u_{n}\right)-\nabla \omega_{\mu, j}\right) \rho_{m}\left(u_{n}\right) d x d t \\
& =\int_{Q} a\left(., T_{k}\left(u_{n}\right), \nabla T_{k}\left(u_{n}\right)\right)\left(\nabla T_{k}\left(u_{n}\right)-\nabla \omega_{\mu, j}\right) d x d t+\int_{u_{n}>k} a\left(., u_{n}, \nabla u_{n}\right)\left(\nabla T_{k}\left(u_{n}\right)-\nabla \omega_{\mu, j}\right) \rho_{m}\left(u_{n}\right) d x d t
\end{aligned}
$$

recall that $\rho_{m}\left(u_{n}\right)=1$ on $\left\{\left|u_{n}\right| \leq k\right\}$.

$$
\text { Let } \begin{aligned}
& s>0, Q_{s}=\left\{(x, t) \in Q:\left|\nabla T_{k}(u)\right| \leq s\right\}, Q_{j}^{s}=\left\{(x, t) \in Q:\left|\nabla T_{k}\left(v_{j}\right)\right| \leq s\right\} . \\
& \int_{Q} a\left(., u_{n}, \nabla u_{n}\right)\left(\nabla T_{k}\left(u_{n}\right)-\nabla \omega_{\mu, j}\right) \rho_{m}\left(u_{n}\right) d x d t \\
& =\int_{Q}\left(a\left(., T_{k}\left(u_{n}\right), \nabla T_{k}\left(u_{n}\right)\right)-a\left(., T_{k}\left(u_{n}\right), \nabla T_{k}\left(v_{j}\right) \mathcal{X}_{j}^{s}\right)\right)\left(\nabla T_{k}\left(u_{n}\right)-\nabla T_{k}\left(v_{j}\right) \mathcal{X}_{j}^{s}\right) d x d t \\
& +\int_{Q} a\left(., T_{k}\left(u_{n}\right), \nabla T_{k}\left(v_{j}\right) \mathcal{X}_{j}^{s}\right)\left(\nabla T_{k}\left(u_{n}\right)-\nabla T_{k}\left(v_{j}\right) \mathcal{X}_{j}^{s}\right) d x d t \\
& +\int_{Q} a\left(., T_{k}\left(u_{n}\right), \nabla T_{k}\left(u_{n}\right)\right) \nabla T_{k}\left(v_{j}\right) \mathcal{X}_{j}^{s} d x d t \\
& -\int_{Q} a\left(., u_{n}, \nabla u_{n}\right) \nabla \omega_{\mu, j} \rho_{m}\left(u_{n}\right) d x d t \\
= & J_{1}+J_{2}+J_{3}+J_{4} .
\end{aligned}
$$

By using the inequality (1.3), we can deduce the existence of some measurable function $h_{k}$ such that 


$$
\begin{gathered}
a\left(., T_{k}\left(u_{n}\right), \nabla T_{k}\left(u_{n}\right)\right) \rightarrow h_{k} \operatorname{in}\left(L_{\bar{M}}(Q)\right)^{N} \text { for } \sigma\left(\prod L_{M}, \prod E_{\bar{M}}\right), \\
J_{2}=\int_{Q} a\left(., T_{k}(u), \nabla T_{k}\left(v_{j}\right) \mathcal{X}_{j}^{s}\right)\left(\nabla T_{k}(u)-\nabla T_{k}\left(v_{j}\right) \mathcal{X}_{j}^{s}\right) d x d t+\varepsilon(n)
\end{gathered}
$$

since

$$
\begin{aligned}
& a\left(., T_{k}\left(u_{n}\right), \nabla T_{k}\left(v_{j}\right) \mathcal{X}_{j}^{s}\right) \rightarrow a\left(., T_{k}(u), \nabla T_{k}\left(v_{j}\right) \mathcal{X}_{j}^{s}\right) \text { strongly in }\left(E_{\bar{M}}(Q)\right)^{N}, \\
& a\left(., T_{k}(u), \nabla T_{k}\left(v_{j}\right) \mathcal{X}_{j}^{s}\right) \rightarrow a\left(., T_{k}(u), \nabla T_{k}(u) \mathcal{X}_{j}^{s}\right) \text { strongly in }\left(E_{\bar{M}}(Q)\right)^{N},
\end{aligned}
$$

and $\nabla T_{k}\left(v_{j}\right) \mathcal{X}_{j}^{s} \rightarrow \nabla T_{k}(u) \mathcal{X}^{s}$ strongly in $\left(L_{\bar{M}}(Q)\right)^{N}$.

Then,

$$
J_{2}=\varepsilon(n, j) .
$$

Following the same way as in $J_{2}$, one has

$$
J_{3}=\int_{Q} h_{k} \nabla T_{k}(u) d x d t+\varepsilon(n, j, \mu, s)
$$

Concerning the terms $J_{4}$ :

$$
\begin{aligned}
J_{4} & =-\int_{Q} a\left(., T_{m+1}\left(u_{n}\right), \nabla T_{m+1}\left(u_{n}\right)\right) \nabla \omega_{\mu, j}^{i} \rho_{m}\left(u_{n}\right) d x d t \\
& =-\int_{\left|u_{n}\right| \leq k} a\left(., T_{m+1}\left(u_{n}\right), \nabla T_{m+1}\left(u_{n}\right)\right) \nabla \omega_{\mu, j} \rho_{m}\left(u_{n}\right) d x d t \\
& -\int_{k<\left|u_{n}\right| \leq m+1} a\left(., T_{m+1}\left(u_{n}\right), \nabla T_{m+1}\left(u_{n}\right)\right) \nabla \omega_{\mu, j} \rho_{m}\left(u_{n}\right) d x d t .
\end{aligned}
$$

Letting $n \rightarrow \infty$, then

$$
J_{4}=-\int_{k<|u| \leq m+1} h_{m+1} \nabla \omega_{\mu, j} \rho_{m}(u) d x d t-\int_{|u| \leq k} h_{k} \nabla \omega_{\mu, j} \rho_{m}(u) d x d t+\varepsilon(n) .
$$

Taking now the limits $j \rightarrow \infty$ and after $\mu \rightarrow \infty$ in the last equality, we obtain

$$
J_{4}=-\int_{Q} h_{k} \nabla T_{k}(u) d x d t+\varepsilon(n, j, \mu) .
$$

Then,

$$
(1)=\int_{Q}\left(a\left(., T_{k}\left(u_{n}\right), \nabla T_{k}\left(u_{n}\right)\right)-a\left(., T_{k}\left(u_{n}\right), \nabla T_{k}\left(v_{j}\right) \mathcal{X}_{j}^{s}\right)\right)\left(\nabla T_{k}\left(u_{n}\right)-\nabla T_{k}\left(v_{j}\right) \mathcal{X}_{j}^{s}\right)+\varepsilon(n, j, \mu, s) .
$$

About (2):

$$
\left|\int_{Q} a\left(., u_{n}, \nabla u_{n}\right) \nabla u_{n}\left(T_{k}\left(u_{n}\right)-\omega_{\mu, j}\right) \rho_{m}^{\prime}\left(u_{n}\right)\right| d x d t \leq C(k) \int_{m<\left|u_{n}\right| \leq m+1} a\left(., u_{n}, \nabla u_{n}\right) \nabla u_{n} d x d t .
$$

Since $\left(u_{n}\right)$ is bounded in $W_{0}^{1, x} L_{D}(Q)$ and using $(i i i)$, we have $\left(a\left(., u_{n}, \nabla u_{n}\right)\right)$ is bounded in $L_{H}(Q)$, then

$$
\left|\int_{m<\left|u_{n}\right| \leq m+1} a\left(., u_{n}, \nabla u_{n}\right) \nabla u_{n} d x d t\right| \leq\left\|a\left(., u_{n}, \nabla u_{n}\right) \mid\right\|_{H, Q}\left\|\nabla u_{n}\right\|_{D, m<\left|u_{n}\right| \leq m+1} \leq \varepsilon(n, m),
$$


so,

$$
(2) \leq \varepsilon(n, m)
$$

About (4):

Since $u \geq \psi$, then $T_{k}(u) \geq T_{k}(\psi)$ and there exist a smooth function $v_{j} \geq T_{k}(\psi)$ such that $v_{j} \rightarrow T_{k}(u)$ for the modular convergence in $W_{0}^{1, x} L_{M}(Q)$.

$$
\text { (4) }=n \int_{Q} T_{n}\left(\left(u_{n}-\psi\right)^{-}\right)\left(T_{k}\left(u_{n}\right)-T_{k}\left(v_{j}\right)_{\mu}\right) \rho_{m}\left(u_{n}\right) d x d t \leq \varepsilon(n, j, \mu) .
$$

Taking into account now the estimation of (1), (2), (4)and (5), we obtain

$$
\int_{Q}\left(a\left(., T_{k}\left(u_{n}\right), \nabla T_{k}\left(u_{n}\right)\right)-a\left(., T_{k}\left(u_{n}\right), \nabla T_{k}\left(v_{j}\right) \mathcal{X}_{j}^{s}\right)\right)\left(\nabla T_{k}\left(u_{n}\right)-\nabla T_{k}\left(v_{j}\right) \mathcal{X}_{j}^{s}\right) d x d t \leq \varepsilon(n, j, \mu, s, m) .
$$

On the other hand,

$$
\begin{aligned}
& \int_{Q}\left(a\left(., T_{k}\left(u_{n}\right), \nabla T_{k}\left(u_{n}\right)\right)-a\left(., T_{k}\left(u_{n}\right), \nabla T_{k}(u) \mathcal{X}^{s}\right)\right)\left(\nabla T_{k}\left(u_{n}\right)-\nabla T_{k}(u) \mathcal{X}^{s}\right) d x d t \\
& -\int_{Q}\left(a\left(., T_{k}\left(u_{n}\right), \nabla T_{k}\left(u_{n}\right)\right)-a\left(., T_{k}\left(u_{n}\right), \nabla T_{k}\left(v_{j}\right) \mathcal{X}_{j}^{s}\right)\right)\left(\nabla T_{k}\left(u_{n}\right)-\nabla T_{k}\left(v_{j}\right) \mathcal{X}_{j}^{s}\right) d x d t \\
& =\int_{Q} a\left(., T_{k}\left(u_{n}\right), \nabla T_{k}\left(u_{n}\right)\right)\left(\nabla T_{k}\left(v_{j}\right) \mathcal{X}_{j}^{s}-\nabla T_{k}(u) \mathcal{X}^{s}\right) d x d t \\
& -\int_{Q} a\left(., T_{k}\left(u_{n}\right), \nabla T_{k}(u) \mathcal{X}^{s}\right)\left(\nabla T_{k}\left(v_{j}\right) \mathcal{X}_{j}^{s}-\nabla T_{k}(u) \mathcal{X}^{s}\right) d x d t \\
& +\int_{Q} a\left(., T_{k}\left(u_{n}\right), \nabla T_{k}\left(v_{j}\right) \mathcal{X}_{j}^{s}\right)\left(\nabla T_{k}\left(u_{n}\right)-\nabla T_{k}\left(v_{j}\right) \mathcal{X}_{j}^{s}\right) d x d t
\end{aligned}
$$

each term of the last right hand side is of the form $\epsilon(n, j, s)$, which gives

$$
\begin{aligned}
& \int_{Q}\left(a\left(., T_{k}\left(u_{n}\right), \nabla T_{k}\left(u_{n}\right)\right)-a\left(., T_{k}\left(u_{n}\right), \nabla T_{k}(u) \mathcal{X}^{s}\right)\right)\left(\nabla T_{k}\left(u_{n}\right)-\nabla T_{k}(u) \mathcal{X}^{s}\right) d x d t \\
& =\int_{Q}\left(a\left(., T_{k}\left(u_{n}\right), \nabla T_{k}\left(u_{n}\right)\right)-a\left(., T_{k}\left(u_{n}\right), \nabla T_{k}\left(v_{j}\right) \mathcal{X}_{j}^{s}\right)\right)\left(\nabla T_{k}\left(u_{n}\right)-\nabla T_{k}\left(v_{j}\right) \mathcal{X}_{j}^{s}\right) d x d t \\
& +(n, j, s) .
\end{aligned}
$$

Following the same technique used by Porretta [2], we have for all $r<s$ :

$$
\int_{Q_{r}}\left(a\left(., T_{k}\left(u_{n}\right), \nabla T_{k}\left(u_{n}\right)\right)-a\left(., T_{k}\left(u_{n}\right), \nabla T_{k}(u)\right)\right)\left(\nabla T_{k}\left(u_{n}\right)-\nabla T_{k}(u)\right) d x d t \rightarrow 0 .
$$

Thus, as in the elliptic case (see [7]), there exists a subsequence also denoted by $u_{n}$ such that

$$
\nabla u_{n} \rightarrow \nabla u \text { a.e. in } Q \text {. }
$$

We deduce then that,

$$
a\left(., T_{k}\left(u_{n}\right), \nabla T_{k}\left(u_{n}\right)\right) \rightarrow a\left(., T_{k}(u), \nabla T_{k}(u)\right) \text { in }\left(L_{\bar{M}}(Q)\right)^{N} \text { for } \sigma\left(\prod L_{M}, \prod E_{\bar{M}}\right)
$$

Lemma 3.7 For all $k>0$,

$$
\nabla T_{k}\left(u_{n}\right) \rightarrow \nabla T_{k}(u) \text { for the modular convergence in }\left(L_{M}(Q)\right)^{N}
$$

\section{Proof:}


We have proved that

$$
\int_{Q}\left(a\left(., T_{k}\left(u_{n}\right), \nabla T_{k}\left(u_{n}\right)\right)-a\left(., T_{k}\left(u_{n}\right), \nabla T_{k}\left(v_{j}\right) \mathcal{X}_{j}^{s}\right)\right)\left(\nabla T_{k}\left(u_{n}\right)-\nabla T_{k}\left(v_{j}\right) \mathcal{X}_{j}^{s}\right) d x d t
$$

$\leq \epsilon(n, j, \mu, s, m)$ (see (3.8)).

We can also deduce that

$$
\begin{aligned}
& \int_{Q}\left(a\left(., T_{k}\left(u_{n}\right), \nabla T_{k}\left(u_{n}\right)\right)-a\left(., T_{k}\left(u_{n}\right), \nabla T_{k}(u) \mathcal{X}^{s}\right)\right)\left(\nabla T_{k}\left(u_{n}\right)-T_{k}(u) \mathcal{X}^{s}\right) d x d t \\
& =\int_{Q}\left(a\left(., T_{k}\left(u_{n}\right), \nabla T_{k}\left(u_{n}\right)\right)-a\left(., T_{k}\left(u_{n}\right), \nabla T_{k}\left(v_{j}\right) \mathcal{X}_{j}^{s}\right)\right)\left(\nabla T_{k}\left(u_{n}\right)-\nabla T_{k}\left(v_{j}\right) \mathcal{X}_{j}^{s}\right) d x d t \\
& +\varepsilon(n, j, s) .
\end{aligned}
$$

Then

$$
\begin{aligned}
& \int_{Q} a\left(., T_{k}\left(u_{n}\right), \nabla T_{k}\left(u_{n}\right)\right) \nabla T_{k}\left(u_{n}\right) d x d t \\
& \leq \int_{Q} a\left(., T_{k}\left(u_{n}\right), \nabla T_{k}\left(u_{n}\right)\right) \nabla T_{k}(u) \mathcal{X}^{s} d x d t \\
& +\int_{Q} a\left(., T_{k}\left(u_{n}\right), \nabla T_{k}(u) \mathcal{X}^{s}\right)\left(\nabla T_{k}\left(u_{n}\right)-T_{k}(u) \mathcal{X}^{s}\right) d x d t+\varepsilon(n, j, \mu, s, m) . \\
& \varlimsup_{n} \int_{Q} a\left(., T_{k}\left(u_{n}\right), \nabla T_{k}\left(u_{n}\right)\right) \nabla T_{k}\left(u_{n}\right) d x d t \leq \int_{Q} a\left(., T_{k}(u), \nabla T_{k}(u)\right) \nabla T_{k}(u) \mathcal{X}^{s} d x d t+\lim _{n} \varepsilon(n, j, \mu, s, m)
\end{aligned}
$$

then,

$$
\varlimsup_{n} \int_{Q} a\left(., T_{k}\left(u_{n}\right), \nabla T_{k}\left(u_{n}\right)\right) \nabla T_{k}\left(u_{n}\right) \leq \int_{Q} a\left(., T_{k}(u), \nabla T_{k}(u)\right) \nabla T_{k}(u) \leq \underline{\lim }_{n} \int_{Q} a\left(., T_{k}\left(u_{n}\right), \nabla T_{k}\left(u_{n}\right)\right) \nabla T_{k}\left(u_{n}\right) .
$$

Letting $n \rightarrow \infty$, we deduce

$$
a\left(., T_{k}\left(u_{n}\right), \nabla T_{k}\left(u_{n}\right)\right) \nabla T_{k}\left(u_{n}\right) \rightarrow a\left(., T_{k}(u), \nabla T_{k}(u)\right) \nabla T_{k}(u) \mathcal{X}^{s} \text { in } L^{1}(Q) .
$$

Using the same argument as above, we obtain

$$
a\left(., T_{k}\left(u_{n}\right), \nabla T_{k}\left(u_{n}\right)\right) \nabla T_{k}\left(u_{n}\right) \rightarrow a\left(., T_{k}(u), \nabla T_{k}(u)\right) \nabla T_{k}(u) \text { in } L^{1}(Q),
$$

and Vitali's theorem and (1.1) gives

$$
\nabla T_{k}\left(u_{n}\right) \rightarrow \nabla T_{k}(u) \text { for the modular convergence in }\left(L_{M}(Q)\right)^{N} .
$$

3.3.1 The convergence of the problems $\left(P_{n}\right)$ and the completion of the proof of Theorem 3.1

The passage to the limit is an easy task by using the last steps, then

$$
a\left(., u_{n}, \nabla u_{n}\right) \rightarrow a(., u, \nabla u) \text { weakly in } L_{H}(Q) \text { and a.e. in } Q,
$$

then,

$$
-\int_{Q} u \frac{\partial \varphi}{\partial t}+\int_{Q} a(., u, \nabla u) \nabla \varphi d x d t-\int_{\Omega} \varphi d u_{0}=\int_{Q} \varphi d \mu,
$$

for all $\phi \in D\left(\mathbf{R}^{N+1}\right)$ which are zero in a neighborhood of $(0, T) \times \partial \Omega$ and $\{T\} \times \Omega$.

\section{Conclusion}

In this article, we have proved the existence of solutions of some class of unilateral problems in the Orlicz-Sobolev spaces when the right-hand side is a Radon measure. 


\section{Competing interests}

The authors declare that they have no competing interests.

Received: 4 April 2011 Accepted: 23 November 2011 Published: 23 November 2011

\section{References}

1. Boccardo, L, Dall'Aglio, A, Gallouet, T, Orsina, L: Nonlinear parabolic equations with measures data. J Funct Anal. 147(1), 237-258 (1997). doi:10.1006/ffan.1996.3040

2. Porretta, A: Existence results for nonlinear parabolic equations via strong convergence of truncations. Ann Mat Pura Appl. IV(CLXXVII), 143-172 (1999)

3. Krasnoselśkii, M, Rutickii, Ya: Convex Functions and Orlicz Spaces. Nodhoff Groningen3 (1969)

4. Adams, R: Sobolev Spaces. Academic Press, New York (1975)

5. Gossez, JP: Some approximation properties in Orlicz-Sobolev spaces. Studia Math. 74, 17-24 (1982)

6. Gossez, JP: Nonlinear elliptic boundary value problems for equations with rapidely or (slowly) increasing coefficients. Trans Am Math Soc. 190, 163-205 (1974)

7. Benkirane, A, Elmahi, A: Almost everywhere convergence of the gradients of solutions to elliptic equations in orlicz spaces and application. Nonlinear Anal TMA. 11(28), 1769-1784 (1997)

8. Talenti, G: Nonlinear elliptic equations, rearrangements of functions and orlicz spaces. Ann Mat Pura Appl. 120(4), 159-184 (1979)

9. Achchab, B, Agouzal, A, Debit, N, Kbiri Alaoui, M, Souissi, A: Nonlinear parabolic inequalities on a general convex. J Math Inequal. 4(2), 271-284 (2010)

10. Kacur, J: Nonlinear Parabolic Boundary Value Problems in the Orlicz-Sobolev Spaces. Partial Differential Equations, Banach Center Publications. PWN Polish Scientific Publishers, Warsaw10 (1983)

11. Gossez, JP, Mustonen, V: Variationnel inequality in orlicz-sobolev spaces. Nonlinear Anal Theory Appl. 11, 379-392 (1987). doi:10.1016/0362-546X(87)90053-8

12. Elmahi, A, Meskine, D: Strongly nonlinear parabolic equations with natural growth terms and $L^{1}$ data in Orlicz spaces. Portugaliae Math 62 (2005). Fas.2

13. Landes, R: On the existence of weak solutions for quasilinear parabolic initial-boundary value problems. Proc R Soc Edinburgh A. 89, 217-137 (1981). doi:10.1017/S0308210500020242

14. Elmahi, A, Meskine, D: Parabolic equations in orlicz space. J Lond Math Soc. 72(2), 410-428 (2005). doi:10.1112/ S0024610705006630

doi:10.1186/1687-2770-2011-46

Cite this article as: Alaoui: Parabolic problems with data measure. Boundary Value Problems 2011 2011:46.

\section{Submit your manuscript to a SpringerOpen ${ }^{\odot}$ journal and benefit from:}

- Convenient online submission

- Rigorous peer review

- Immediate publication on acceptance

- Open access: articles freely available online

- High visibility within the field

- Retaining the copyright to your article

Submit your next manuscript at $\gg$ springeropen.com 